TITLE:

\title{
Nonlinear temperature dependence of resistivity in single crystal Ag5Pb2O6
}

$\operatorname{AUTHOR}(S)$ :

Yonezawa, S; Maeno, Y

\section{CITATION:}

Yonezawa, S ...[et al]. Nonlinear temperature dependence of resistivity in single crystal Ag5Pb206. PHYSICAL REVIEW B 2004, 70(18): 184523.

ISSUE DATE:

2004-11

URL:

http://hdl.handle.net/2433/50044

RIGHT:

Copyright 2004 American Physical Society 
PHYSICAL REVIEW B 70, 184523 (2004)

\title{
Nonlinear temperature dependence of resistivity in single crystal $\mathrm{Ag}_{5} \mathrm{~Pb}_{2} \mathrm{O}_{6}$
}

\author{
Shingo Yonezawa ${ }^{1, *}$ and Yoshiteru Maeno ${ }^{1,2}$ \\ ${ }^{1}$ Department of Physics, Graduate School of Science, Kyoto University, Kyoto, 606-8502, Japan \\ ${ }^{2}$ International Innovation Center, Kyoto University, Kyoto, 606-8501, Japan \\ (Received 14 May 2004; revised manuscript received 18 August 2004; published 22 November 2004)
}

\begin{abstract}
We measured electrical resistivity, specific heat and magnetic susceptibility of single crystals of highly conductive oxide $\mathrm{Ag}_{5} \mathrm{~Pb}_{2} \mathrm{O}_{6}$, which has a layered structure containing a Kagome lattice. Both the out-of-plane and in-plane resistivity show $T^{2}$ dependence in an unusually wide range of temperatures up to room temperature. This behavior cannot be accounted for either by electron correlation or by electron-phonon scattering with high frequency optic phonons. In addition, a phase transition with a large diamagnetic signal was found in the ac susceptibility, which strongly suggests the existence of a superconducting phase below $48 \mathrm{mK}$.
\end{abstract}

DOI: 10.1103/PhysRevB.70.184523

\section{INTRODUCTION}

One of the central issues of solid state physics in recent decades has been the physics of an electron-electron (e-e) correlation. As a hallmark of the Fermi liquid state formed by strong electron correlations, the resistivity exhibits $T^{2}$ dependence at low temperatures, as in many heavy-fermion systems. ${ }^{1}$ The $T^{2}$ dependence of resistivity also appears in quasi-2-dimensional Fermi liquid of oxides such as $\mathrm{Sr}_{2} \mathrm{RuO}_{4},{ }^{2}$ which is strongly suggested as a spin-triplet superconductor with $T_{\mathrm{c}}=1.5 \mathrm{~K}$. Interestingly, a layered compound $\mathrm{MgB}_{2}$, which shows superconductivity at $T_{\mathrm{c}}=39 \mathrm{~K},{ }^{3}$ also exhibits $T^{2}$ dependence in the resistivity up to $240 \mathrm{~K} .{ }^{4,5}$ The $\rho \propto T^{2}$ law in $\mathrm{MgB}_{2}$ is attributed, not to the e-e correlation but to the strong electron-phonon (e-ph) coupling between a quasi-2-dimensional conduction band and an optic phonon mode in the boron honeycomb layer. ${ }^{6}$

As another example of a layered system which exhibits $T^{2}$ resistivity, but in a much wider temperature range up to room temperature, we report here the physical properties of a layered oxide $\mathrm{Ag}_{5} \mathrm{~Pb}_{2} \mathrm{O}_{6}$. This material was first reported by Byström and Evers in 1950. ${ }^{7}$ As shown in Fig. 1, the crystal structure consists of an alternating stacking of Kagome layers of silver and honeycomb-like networks of $\mathrm{PbO}_{6}$ octahedra, which is connected by one-dimensional chains of silver penetrating the layers. As a whole, $\mathrm{Ag}_{5} \mathrm{~Pb}_{2} \mathrm{O}_{6}$ forms a hexagonal $P \overline{3} 1 \mathrm{~m}$ structure with lattice parameters $a=b$ $=5.9324(3) \AA$ and $c=6.4105(4) \AA{ }^{8}$

Far before the report of the $\rho \propto T^{2}$ law, it was suggested by Dickens that $\mathrm{Ag}_{5} \mathrm{~Pb}_{2} \mathrm{O}_{6}$ shows metallic conductivity, ${ }^{9}$ and it was confirmed by Jansen et al. in polycrystalline samples. ${ }^{8}$ Band calculation by Brennan et al. revealed that its Fermi level is located near the center of the band and that its conductivity is attributed to one free electron from one formula unit, de-localized to all over the silver substructure; thus its valence formula is represented as $\left(\mathrm{Ag}_{5}\right)^{4+}\left(\mathrm{Pb}^{4+}\right)_{2}\left(\mathrm{O}^{2-}\right)_{6}{ }^{10}$ The $T^{2}$ dependence of the resistivity up to room temperature for the out-of-plane resistivity of single crystals was recently reported by Shibata et al. at meetings of the Physical Society of Japan. ${ }^{11}$ This dependence is rather unusual, because resistivity of almost all metals show $T$-linear dependence around room temperature due to the dominance of e-ph scattering.
PACS number(s): 74.70.Dd, 72.15.Qm, 72.80.Ga
It was Jansen et al. who first succeeded in the controlled synthesis of polycrystalline $\mathrm{Ag}_{5} \mathrm{~Pb}_{2} \mathrm{O}_{6}$ by a high oxygen pressure method. ${ }^{8} \mathrm{~A}$ recent report by $\mathrm{H}$. Abe et al. on a new method to obtain single crystals ${ }^{12}$ made it possible to perform more precise experiments. Nevertheless, so far no article on experiments with single crystals has been published, although there are several articles on closely related materials, such as $\mathrm{Bi}$ and In substitution to the $\mathrm{Pb}$ site, ${ }^{13} \mathrm{Cu}$ substitution to the $\mathrm{Pb}$ site ${ }^{14}$ and $\mathrm{Ag}_{5} \mathrm{~Pb}_{2} \mathrm{O}_{6}$ with defects. ${ }^{15} \mathrm{We}$ note that there are also articles on powder samples by Djurek et al. reporting several strange properties, ${ }^{16,17}$ which have not been reproduced by other groups to the best of our knowledge.

We present here the first report on physical properties of single crystalline $\mathrm{Ag}_{5} \mathrm{~Pb}_{2} \mathrm{O}_{6}$. We synthesized the single crystals and measured resistivity, specific heat and magnetic susceptibility. In resistivity, we succeeded not only in reproducing the $T^{2}$ dependence of the out-of-plane resistivity, but also in finding the same dependence of the in-plane resistivity for the first time. We will discuss the possible origin of the unusual $T^{2}$ resistivity. What is more, we found a large signal of diamagnetic phase transition around $48 \mathrm{mK}$, which implies that a bulk superconducting phase exists in $\mathrm{Ag}_{5} \mathrm{~Pb}_{2} \mathrm{O}_{6}$.

\section{EXPERIMENTS}

The single crystals of $\mathrm{Ag}_{5} \mathrm{~Pb}_{2} \mathrm{O}_{6}$ used in this study were grown by basically the same method as the one Shibata et al. reported. ${ }^{11} \mathrm{As}$ starting materials, we used $\mathrm{AgNO}_{3}$ (99.9999\%) and $\mathrm{Pb}\left(\mathrm{NO}_{3}\right)_{2}(99.999 \%)$. We ground them in a mortar, and put the powder into thin $\mathrm{Al}_{2} \mathrm{O}_{3}$ crucibles with a $10-\mathrm{mm}$ inner diameter. The ratio of $\mathrm{Ag}$ and $\mathrm{Pb}$ is basically stoichiometric. The crucibles were heated in air to $80^{\circ} \mathrm{C}$ and kept for 3 hours, then heated up to $300^{\circ} \mathrm{C}$ in 8 hours and up to the reaction temperature at $394{ }^{\circ} \mathrm{C}$ in another 5 hours. We note here that one should cover the crucibles with larger crucibles or something in order to avoid pollution of the furnace, since melting $\mathrm{AgNO}_{3}$ sometimes splashes out of the crucibles. The temperature was kept at this temperature for 48 hours, and the crucibles were furnace-cooled to room temperature. ${ }^{11}$ 


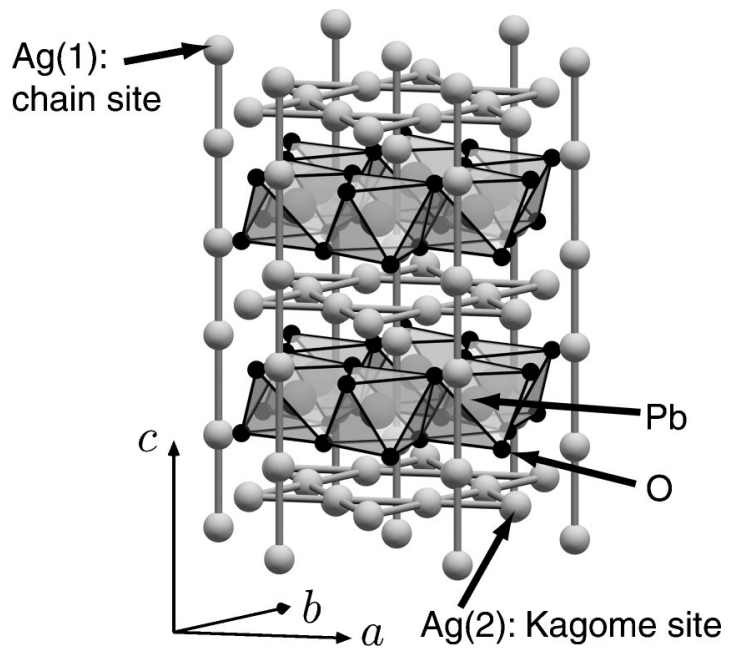

FIG. 1. Crystal structure of $\mathrm{Ag}_{5} \mathrm{~Pb}_{2} \mathrm{O}_{6}$.

However, the crystals obtained with the above parameters do not have well-defined shapes as shown in Fig. 2(a), thus they are not suitable for resistivity measurements. We found that we can grow better-shaped crystals by choosing lower reaction temperatures. Furthermore, we also discovered that adding extra $\mathrm{AgNO}_{3}$ promotes the growth, because $\mathrm{AgNO}_{3}$, which melts at $212^{\circ} \mathrm{C}$, apparently plays a role of self-flux. Figure 2(b) shows the crystals grown with twice the amount of $\mathrm{AgNO}_{3}$ and with a reaction at $380{ }^{\circ} \mathrm{C}$. Using this process, we obtained crystals in the shape of hexagonal sticks, which are shown in Figs. 2(c) and 2(d). The long axis of the sticks are along the $c$ axis. The largest size of crystals was approximately $1 \mathrm{~mm}$ in length and $0.5 \mathrm{~mm}$ in thickness.

For characterization of the obtained crystals, we used powder x-ray diffractometry with $\mathrm{CuK} \alpha_{1}$ radiation, which

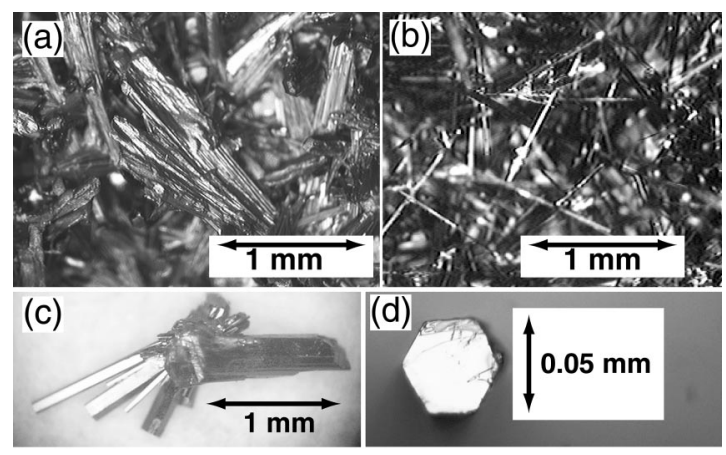

FIG. 2. Photos of single crystals of $\mathrm{Ag}_{5} \mathrm{~Pb}_{2} \mathrm{O}_{6}$ taken with optical microscopes. The long axis of these crystals are along the $c$ axis. (a) Single crystals in a crucible which are obtained with basic reaction parameters described in the text. (b) Single crystals at the bottom of a crucible when we mixed $\mathrm{AgNO}_{3}$ and $\mathrm{Pb}\left(\mathrm{NO}_{3}\right)_{2}$ with a ratio of $\mathrm{Ag}: \mathrm{Pb}=5: 1$ and also chose a lower reaction temperature $\left(380^{\circ} \mathrm{C}\right)$. The crystals grew dispersed from each other and the shapes became better with the aid of self-flux $\mathrm{AgNO}_{3}$. In this case $\mathrm{AgNO}_{3}$ little remained since most of unreacted $\mathrm{AgNO}_{3}$ had been evaporated or resolved into Ag metal. (c) A group of single crystals removed from the crucible in photo (b). We obtained a lot of crystals with a perfectly hexagonal-stick shape by adding self-flux $\mathrm{AgNO}_{3}$. (d) A cross section of a typical single crystal (not the largest). This hexagonal plane is in the $a b$ plane of $\mathrm{Ag}_{5} \mathrm{~Pb}_{2} \mathrm{O}_{6}$.

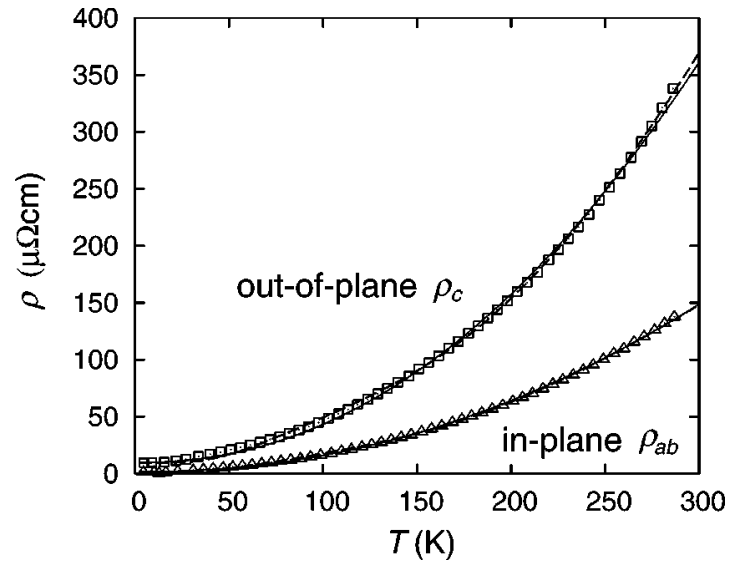

FIG. 3. Temperature dependence of the out-of-plane (open squares) and the in-plane resistivity (open triangles) down to $3 \mathrm{~K}$. Solid lines are the fitting with $\rho(T)=\rho_{0}+A^{\prime} T^{p}$ with $p=2.13$ for $\rho_{c}$ and $p=2.11$ for $\rho_{a b}$. Dashed lines are the fitting assuming the existence of an Einstein-like optic phonon mode.

confirmed that these crystals were single-phased $\operatorname{Ag}_{5} \mathrm{~Pb}_{2} \mathrm{O}_{6}$. Resistivity was measured using a standard four-probe method with dc or ac current. Specific heat was measured with a commercial calorimeter (Quantum Design, model PPMS), and dc susceptibility by a SQUID magnetometer (Quantum Design, model MPMS). The ac susceptibility was measured by a mutual inductance method down to $38 \mathrm{mK}$ using a ${ }^{4} \mathrm{He}-{ }^{3} \mathrm{He}$ dilution refrigerator (Cryoconcept, model DR-JT-S-100-10).

We note here about the lowest temperature of the resistivity measurement. We used an ultrasonic soldering method to attach the leads to the crystals, because several conductive pastes (e.g., silver paste) we used all damaged the sample seriously. On the other hand, the solder we used (Cerasolzer \#123, Senju Metal Industry) becomes superconducting below $3 \mathrm{~K}$, which makes it impossible for us to measure the sample resistivity accurately below $3 \mathrm{~K}$. We should also note that the accuracy of in-plane resistivity is less reliable than that of the out-of-plane resistivity. This is because we could not shape up the samples before attaching leads to the crystal. Thus the electric current did not flow uniformly and we could not determine $\rho$ by the simple relation as $\rho=R(S / l)$, where $S$ is the cross section and $l$ is the lead spacing. The results of $\rho_{a b}$ shown in Fig. 3 is an estimation assuming uniform current flow. Nevertheless, the relative variation of $\rho$ in temperature should be reliable.

\section{RESULTS}

Figure 3 shows the resistivity along the $c$ axis $\left(\rho_{c}\right)$ and in the $a b$ plane $\left(\rho_{a b}\right)$. Anisotropy of resistivity at $280 \mathrm{~K}$ is rather small and approximately 2 , which is consistent with the results of the band calculation by Brennan et al. ${ }^{10}$ The residual resistivity for $\rho_{c}$ and $\rho_{a b}$ are $9.7 \mu \Omega \mathrm{cm}$ and $1.5 \mu \Omega \mathrm{cm}$, respectively; this means residual resistivity ratios (RRR) are 35 for $\rho_{c}$ and 93 for $\rho_{a b}$, which show that the quality of the samples was quite good. If we assume its temperature dependence as $\rho(T)=\rho_{0}+A^{\prime} T^{p}$, we obtained the ex- 


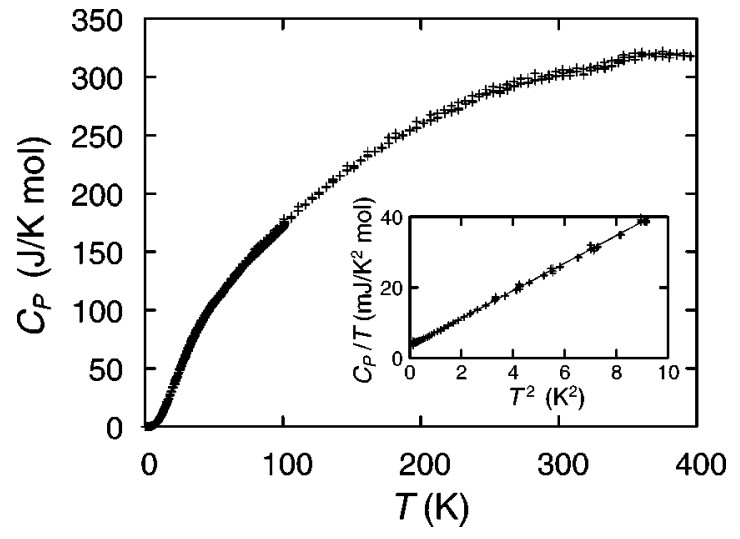

FIG. 4. Specific heat of $\mathrm{Ag}_{5} \mathrm{~Pb}_{2} \mathrm{O}_{6}$ down to $0.35 \mathrm{~K}$. These figures contain the results of several different samples. Inset: $C_{P} / T$ plotted against $T^{2}$ below $3.3 \mathrm{~K}$. Solid line is the fitting $C_{P}(T) / T$ $=\gamma_{\mathrm{e}}+\beta_{\mathrm{p}} T^{2}$ with $\gamma_{\mathrm{e}}=3.42 \mathrm{~mJ} / \mathrm{K}^{2} \mathrm{~mol}$ and $\beta_{\mathrm{p}}=3.91 \mathrm{~mJ} / \mathrm{K}^{4} \mathrm{~mol}$.

ponent as $p=2.13$ for $\rho_{c}$ and $p=2.11$ for $\rho_{a b}$ from the fitting in the all temperature range. ${ }^{18}$ For both directions, $p$ is close to 2 ; thus if we approximate as $\rho(T)=\rho_{0}+A T^{2}$, we obtained the coefficient of $T^{2}$ term $A_{c}=3.78 \times 10^{-3} \mu \Omega \mathrm{cm} / \mathrm{K}^{2}$ for $\rho_{c}(T)$ and $A_{a b}=1.6 \times 10^{-3} \mu \Omega \mathrm{cm} / \mathrm{K}^{2}$ for $\rho_{a b}(T)$.

The measured specific heat is shown in Fig. 4. Since $\mathrm{Ag}_{5} \mathrm{~Pb}_{2} \mathrm{O}_{6}$ does not contain any magnetic ions, we express $C_{P}$ in a 1-mol formula unit, in other words, of 1-mol conduction electrons. In the high temperature region, $C_{P}$ converges to a constant value, which agrees with the DulongPetit classical phonon specific heat, $C_{\mathrm{cl}}=3 k_{\mathrm{B}} r N_{\mathrm{A}}$ $=324 \mathrm{~J} / \mathrm{K} \mathrm{mol},{ }^{19}$ where $r=13$ is the number of atoms in one formula unit. We also measured the specific heat in magnetic fields, but there was no difference between the data in $0 \mathrm{~T}$ and in $7 \mathrm{~T}$. The inset is the $C_{P} / T$-vs- $T^{2}$ plot below $3.3 \mathrm{~K}$. We obtained the electronic specific heat coefficient $\gamma_{\mathrm{e}}$ $=3.42 \mathrm{~mJ} / \mathrm{K}^{2} \mathrm{~mol}$ and the Debye temperature $\Theta_{\mathrm{D}}=186 \mathrm{~K}$ from this plot.

$\mathrm{Ag}_{5} \mathrm{~Pb}_{2} \mathrm{O}_{6}$ displays weak constant diamagnetism in $10 \mathrm{~K}<T<350 \mathrm{~K},(-2.07 \pm 0.02) \times 10^{-4} \mathrm{emu} / \mathrm{mol}$, from the dc susceptibility measurement. Below $10 \mathrm{~K}, \chi_{\mathrm{dc}}$ increases probably owing to paramagnetic impurities. To subtract the diamagnetic contribution from the ion cores, we used the values of the susceptibility of cores in the literature ${ }^{20}$ we estimated the total contribution from the cores as $-2.44 \times 10^{-4} \mathrm{emu} / \mathrm{mol}$. When we subtracted this value from the experimental value, we obtained $(+3.7 \pm 0.2)$ $\times 10^{-5} \mathrm{emu} / \mathrm{mol}$. This is the Pauli susceptibility of conduction electrons because there are no magnetic ions in $\mathrm{Ag}_{5} \mathrm{~Pb}_{2} \mathrm{O}_{6}$.

In the ac susceptibility measurement, we found a very large signal around $45 \mathrm{mK}$ as shown in Fig. 5. The signal was reproducible in different samples, in different susceptibility cells and in different ac magnetic field amplitudes and frequencies. We also measured ac susceptibility of a bulk pure Al with approximately the same volume as the measured $\mathrm{Ag}_{5} \mathrm{~Pb}_{2} \mathrm{O}_{6}$ using the same cell, and found that $\mathrm{Ag}_{5} \mathrm{~Pb}_{2} \mathrm{O}_{6}$ yields as large a diamagnetic signal as that of the superconducting transition of Al. Moreover, this transition disappears under a few Oe magnetic field, and $\mathrm{Ag}_{5} \mathrm{~Pb}_{2} \mathrm{O}_{6}$

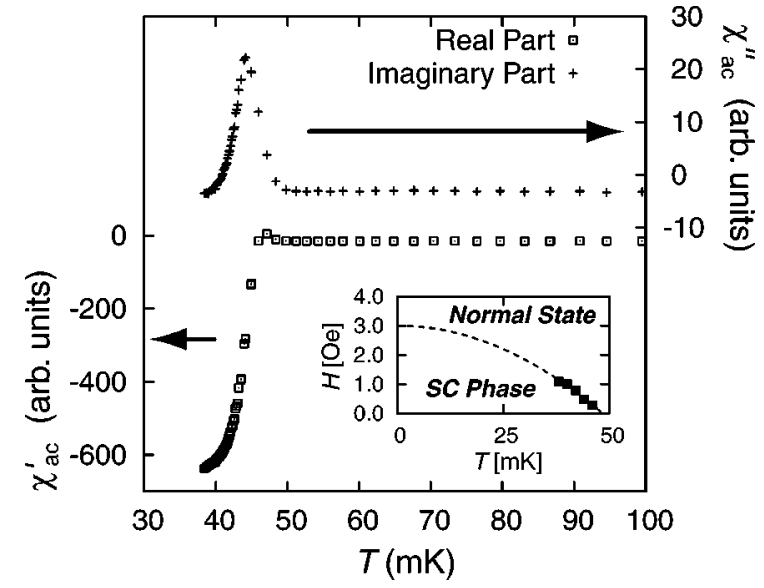

FIG. 5. The ac magnetic susceptibility below $0.1 \mathrm{~K}$, measured with an ac magnetic field of amplitude $2.3 \times 10^{-2}$ Oe and frequency $887 \mathrm{~Hz}$. A very large diamagnetic phase transition signal was found below $45 \mathrm{mK}$, which suggests the existence of a superconducting (SC) phase. Inset: Phase diagram of $\mathrm{Ag}_{5} \mathrm{~Pb}_{2} \mathrm{O}_{6}$ below $50 \mathrm{mK}$. Closed squares are " $H_{\mathrm{c}}$ " determined from the peaks of imaginary part $\chi_{\mathrm{ac}}^{\prime \prime}$. The dashed line shows the classical parabolic law of superconductivity: $H_{\mathrm{c}}(T)=H_{\mathrm{c} 0}\left[1-\left(T / T_{\mathrm{c} 0}\right)^{2}\right]$.

does not contain any magnetic ions which may cause a diamagnetic transition. Thus this signal strongly implies the existence of a bulk superconducting phase of $\mathrm{Ag}_{5} \mathrm{~Pb}_{2} \mathrm{O}_{6}$. The inset of Fig. 5 is the phase diagram of this new phase. " $H_{\mathrm{c}}(T)$ " was determined from the peaks of imaginary part $\chi_{\mathrm{ac}}^{\prime \prime}$ by sweeping the magnetic field at each fixed temperature. We note that values of $H$ in the inset of Fig. 5 are shifted because exact zero point of our magnet changed by a few Oe due to the residual magnetic field. Thus we swept magnetic field from +10 Oe to -10 Oe and determined the exact zero point from the symmetry of the $\chi_{\mathrm{ac}}$ data. " $H_{\mathrm{c}}$ " is well fitted by a parabolic law for superconductor: $H_{\mathrm{c}}(T)$ $=H_{\mathrm{c} 0}\left[1-\left(T / T_{\mathrm{c} 0}\right)^{2}\right]$ where $H_{\mathrm{c} 0}=3.0$ Oe and $T_{\mathrm{c} 0}=48 \mathrm{mK}$ obtained by fitting. This variation also supports the evidence of superconductivity.

\section{DISCUSSION}

Now we discuss the origin of the $T^{2}$ dependence of resistivity. One possibility is e-e interaction. The resistivity of materials in which a strong e-e correlation exists shows $T^{2}$ dependence only at a temperature much lower than room temperature, because the characteristic temperature of manybody Fermi liquid formation is low and also the $T^{2}$-behavior requires the dominance of the e-e scattering over the e-ph scattering. If the $\rho \propto T^{2}$ law up to room temperature of $\mathrm{Ag}_{5} \mathrm{~Pb}_{2} \mathrm{O}_{6}$ comes from the e-e correlation, the e-e correlation must be so large as to dominate over the e-ph scattering even at room temperature. But it seems impossible to expect a strong e-e interaction from the discussions below. First, the electronic specific heat coefficient of $\mathrm{Ag}_{5} \mathrm{~Pb}_{2} \mathrm{O}_{6}$ is comparable to those of normal metals and 1-3 orders of magnitude smaller than those of strongly-correlated electron systems. Moreover, neither the Wilson ratio nor the Kadowaki-Woods $(\mathrm{K}-\mathrm{W})$ ratio shows evidence of strong e-e correlations. The 
Wilson ratio, which is given by $\left(\pi^{2} k_{\mathrm{B}}^{2} / 3 \mu_{\mathrm{B}}^{2}\right)\left(\chi_{\text {Pauli }} / \gamma_{\mathrm{e}}\right)$, is expected to increase from unity to 2 as the e-e interaction increases from 0 to $\infty .{ }^{21}$ In $\mathrm{Ag}_{5} \mathrm{~Pb}_{2} \mathrm{O}_{6}$, the Wilson ratio is 0.79 , close to unity, implying that the e-e interaction is weak. Of course we should note that the value of $\chi_{\text {Pauli }}$ may not be very accurate because the subtracted core contribution is much larger than $\chi_{\text {Pauli }}$. The $\mathrm{K}-\mathrm{W}$ ratio is defined as $A / \gamma_{\mathrm{e}}^{2}$ and becomes a universal value $a_{0}=1.0$ $\times 10^{-5} \mu \Omega \mathrm{cm}(\mathrm{K} \mathrm{mol} / \mathrm{mJ})^{2}$ in most strongly-correlated systems. ${ }^{1,22}$ The K-W ratio of $\mathrm{Ag}_{5} \mathrm{~Pb}_{2} \mathrm{O}_{6}$ is $32.3 a_{0}$ for $\rho_{c}$ and approximately $14 a_{0}$ for $\rho_{a b}$; both are much larger than $a_{0}$. These deviation imply that $\mathrm{Ag}_{5} \mathrm{~Pb}_{2} \mathrm{O}_{6}$ cannot be classified into strongly-correlated electron systems and that the $T^{2}$ dependence of $\rho$ is not due to the e-e interaction.

Another possible origin is the e-ph scattering. Basically, resistivity governed by the e-ph interaction is $T$-linear above $\Theta_{\mathrm{D}} / 5(\approx 20-100 \mathrm{~K}$, in most metals). But there are some materials of which resistivity shows nonlinear $T$ dependence at a relatively high temperature because of the e-ph scattering by high energy optic phonons. For example, $\mathrm{MgB}_{2}$ shows $\rho \propto T^{2}$ behavior in $T_{\mathrm{c}}<T \leqq 240 \mathrm{~K}, 4,5$ and $\mathrm{ReO}_{3}$ exhibits an unusual curvature in the resistivity in $120 \mathrm{~K} \lesssim T \leqq 220 \mathrm{~K}^{23}$ When analyzing nonlinear $T$ dependence of resistivity in these materials, it is a common approach to assume the optic phonons as Einstein-like. ${ }^{6,23,24}$ In this method, the Boltzmann equation is a starting point:

$$
\rho(T)=\rho_{0}+\frac{(4 \pi)^{2} k_{\mathrm{B}} T}{\hbar \omega_{\mathrm{p}}^{2}} \int_{0}^{\omega_{\max }} \frac{d \omega}{\omega} \alpha^{2} F(\omega)\left(\frac{x}{\sinh x}\right)^{2},
$$

where $x=\hbar \omega / 2 k_{\mathrm{B}} T, \alpha^{2} F(\omega)$ is the Eliashberg function, and $\omega_{\mathrm{p}}$ is the plasma frequency of conduction electrons. We assume that we can separate the Eliashberg function into the Debye term and the Einstein term:

$$
\alpha^{2} F(\omega)=2 \lambda_{\mathrm{D}}\left(\frac{\omega}{\Omega_{\mathrm{D}}}\right)^{4} \theta\left(\Omega_{\mathrm{D}}-\omega\right)+\frac{\lambda_{\mathrm{E}} \Omega_{\mathrm{E}}}{2} \delta\left(\omega-\Omega_{\mathrm{E}}\right),
$$

where $\Omega_{\mathrm{D}}$ and $\Omega_{\mathrm{E}}$ are the Debye frequency and the frequency of Einstein phonons, respectively, and $\lambda_{D}$ and $\lambda_{E}$ are the e-ph coupling constant of Debye phonons and of Einstein phonons, respectively. Now we can substitute this Eliashberg function into Eq. (1), and fit it with the experimental data. When we used $k_{\mathrm{B}} \Omega_{\mathrm{D}} / \hbar, k_{\mathrm{B}} \Omega_{\mathrm{E}} / \hbar, \lambda_{\mathrm{D}} / \omega_{\mathrm{p}}^{2}$ and $\lambda_{\mathrm{E}} / \omega_{\mathrm{p}}^{2}$ as fitting parameters, we obtained $k_{\mathrm{B}} \Omega_{\mathrm{D}} / \hbar=290 \mathrm{~K}$ and $k_{\mathrm{B}} \Omega_{\mathrm{E}} / \hbar$ $=1200 \mathrm{~K}$ from the fitting with $\rho_{c}$ data, and $k_{\mathrm{B}} \Omega_{\mathrm{D}} / \hbar=280 \mathrm{~K}$ and $k_{\mathrm{B}} \Omega_{\mathrm{E}} / \hbar=1100 \mathrm{~K}$ from $\rho_{a b}$. The results of the fittings are shown in Fig. 3 with dashed lines. But the existence of such a high frequency Einstein-like phonon is doubtful. This is because specific heat shown in Fig. 4 converges to the Dulong-Petit value around $350 \mathrm{~K}$, implying that all phonon modes are "freely" excited at this temperature and thus there seems to be no more phonon mode with energy much larger than $350 \mathrm{~K} \cdot{ }^{25}$ For comparison, the specific heat of $\mathrm{MgB}_{2}$, in which the optic phonon mode with a wave number of $620 \mathrm{~cm}^{-1}$ (890 K in energy) plays important roles, is indeed not at all saturated even at $300 \mathrm{~K} .{ }^{26}$

If we assume that the novel phase below $48 \mathrm{mK}$ is a phonon-mediated superconducting phase, we can estimate the e-ph coupling constant $\lambda$ from McMillan's relation: ${ }^{27}$

$$
T_{\mathrm{c}}=\frac{\Theta_{\mathrm{D}}}{1.45} \exp \left[-\frac{1.04(1+\lambda)}{\lambda-\mu^{*}(1+0.62 \lambda)}\right] .
$$

When we assumed the psudopotential as $\mu^{*}=0.1$ and used $\Theta_{\mathrm{D}}=186 \mathrm{~K}$, we obtained $\lambda=0.288$, which is comparable to those of $\mathrm{W} \quad\left(\lambda=0.28, T_{\mathrm{c}}=12 \mathrm{mK}\right)$ or Be $\left(\lambda=0.23, T_{\mathrm{c}}\right.$ $=26 \mathrm{mK}) \cdot{ }^{27}$ Such a small $\lambda$ may be another evidence that a strong e-ph interaction does not exist in $\mathrm{Ag}_{5} \mathrm{~Pb}_{2} \mathrm{O}_{6}$.

The unusual $\rho \propto T^{2}$ behavior up to room temperature thus can be attributed to neither an e-e interaction nor an e-ph interaction including the optic phonons. We note here that a one-dimensional chain organic compound TTF-TCNQ exhibits a $T^{2.33}$ dependence of resistivity in $60 \mathrm{~K}<T<300$ $\mathrm{K},{ }^{28}$ attributable to the librational phonon mode in the chain. ${ }^{29}$ This scenario is not applicable to $\mathrm{Ag}_{5} \mathrm{~Pb}_{2} \mathrm{O}_{6}$ because of the rigidness of the lattice against twisting motion of the silver chain. Nevertheless, a unique phonon mode may exist also in $\mathrm{Ag}_{5} \mathrm{~Pb}_{2} \mathrm{O}_{6}$ which gives rise to the $T^{2}$ dependence. Thus a detailed investigation into the phonon modes in $\mathrm{Ag}_{5} \mathrm{~Pb}_{2} \mathrm{O}_{6}$ and into their strength of coupling with conduction electrons may elucidate the $T^{2}$ dependence.

\section{CONCLUSION}

In conclusion, we synthesized single crystals of $\mathrm{Ag}_{5} \mathrm{~Pb}_{2} \mathrm{O}_{6}$ and measured their resistivity, specific heat and magnetic susceptibility. The resistivity exhibits unusual $T^{2}$ dependence up to room temperature both along the $c$ axis and in the $a b$ plane. This behavior cannot be explained by an e-e interaction. It seems also impossible to explain the $T^{2}$ dependence from the e-ph scattering by optic phonons. Thus there should be other scattering mechanisms in $\mathrm{Ag}_{5} \mathrm{~Pb}_{2} \mathrm{O}_{6}$. Moreover, a phase transition with a large diamagnetic signal was found below $48 \mathrm{mK}$ and below 3 Oe. This new phase seems to be a bulk superconducting phase in $\mathrm{Ag}_{5} \mathrm{~Pb}_{2} \mathrm{O}_{6}$.

\section{ACKNOWLEDGMENTS}

We would like to thank Prof. Y. Yamada at Niigata University, K. Deguchi and K. Kitagawa at Kyoto University for their great help for measurements and their useful discussions. We also acknowledge Prof. K. Tanaka and Y. Okuda at Kyoto University for the Raman spectroscopy and S. Fujimoto at Kyoto University for his helpful suggestions. This work is supported by a Grant-in-Aid for the 21st Century COE "Center for Diversity and Universality in Physics" from MEXT of Japan. It is also in part supported by additional Grants-in-Aid from JSPS and MEXT. 
*Electronic address: yonezawa@scphys.kyoto-u.ac.jp

${ }^{1}$ K. Kadowaki and S. B. Woods, Solid State Commun. 58, 507 (1986).

${ }^{2}$ Y. Maeno, K. Yoshida, H. Hashimoto, S. Nishizaki, S. ichi Ikeda, M. Nohara, T. Fujita, A. P. Mackenzie, N. E. Hussey, J. G. Bednorz et al., J. Phys. Soc. Jpn. 66, 1405 (1997).

${ }^{3}$ J. Nagamatsu, N. Nakagawa, T. Muranaka, Y. Zenitani, and J. Akimitsu, Nature (London) 410, 63 (2001).

${ }^{4}$ M. Xu, H. Kitazawa, Y. Takano, J. Ye, K. Nishida, H. Abe, A. Matsushita, N. Tsujii, and G. Kido, Appl. Phys. Lett. 79, 2779 (2001).

${ }^{5}$ K. H. P. Kim, J.-H. Choi, C. U. Jung, P. Chowdhury, H.-S. Lee, M.-S. Park, H.-J. Kim, J. Y. Kim, Z. Du, E.-M. Choi et al., Phys. Rev. B 65, 100510(R) (2002).

${ }^{6}$ T. Masui, K. Yoshida, S. Lee, A. Yamamoto, and S. Tajima, Phys. Rev. B 65, 214513 (2002).

${ }^{7}$ A. Byström and L. Evers, Acta Chem. Scand. (1947-1973) 4, 613 (1950).

${ }^{8}$ M. Jansen, M. Bortz, and K. Heidebrecht, J. Less-Common Met. 161, 17 (1990).

${ }^{9}$ B. Dickens, J. Inorg. Nucl. Chem. 28, 613 (1966).

${ }^{10}$ T. D. Brennan and J. K. Burdett, Inorg. Chem. 33, 4794 (1994).

${ }^{11}$ T. Shibata, Y. Yamada, A. Matsushita, H. Abe, and S. Kubo, presented at the Physical Society of Japan (JPS) fall meeting in 2001 and the spring meeting in 2002; T. Shibata, Y. Yamada, A. Matsushita, and S. Kubo, presented at the JPS fall meeting in 2002.

${ }^{12}$ H. Abe, J. Ye, M. Imai, K. Yoshii, and A. Matsushita, J. Cryst. Growth 241, 347 (2002).

${ }^{13}$ M. Bortz, M. Jansen, H. Hohl, and E. Bucher, J. Solid State Chem. 103, 447 (1993).
${ }^{14}$ E. M. Tejada-Rosales, J. Oró-solé, and P. Gómez-Romero, J. Solid State Chem. 163, 151 (2002).

${ }^{15}$ K. Iwasaki, H. Yamane, S. Kubota, J. Takahashi, Y. Miyazaki, T. Kajitani, K. Nakajima, T. Yamashita, and M. Shimada, Physica C 382, 263 (2002).

${ }^{16}$ D. Djurek, Z. Medunić, M. Paljevic, and A. Tonejc, Phys. Status Solidi A 201, 544 (2004).

${ }^{17}$ D. Djurek, Z. Medunić, M. Paljevic, and A. Tonejc, cond-mat/ 0403027, 2004.

${ }^{18}$ The systematic correction due to thermal expansion at higher temperatures gives a negligible change in the $p$.

${ }^{19}$ Strictly speaking, $C_{\mathrm{cl}}$ is the specific heat at constant volume while $C_{P}$ is at constant pressure; however the difference of them should be small.

${ }^{20} \mathrm{P}$. W. Selwood, Magnetochemistry (Interscience, New York, 1956).

${ }^{21}$ K. Yamada, Prog. Theor. Phys. 53, 970 (1975).

${ }^{22}$ K. Miyake and T. Matsuura, Solid State Commun. 71, 1149 (1989).

${ }^{23}$ C. N. King, H. C. Kirsch, and T. H. Geballe, Solid State Commun. 9, 907 (1971).

${ }^{24}$ P. B. Allen and W. W. Schulz, Phys. Rev. B 47, 14434 (1993).

${ }^{25}$ Laser Raman spectra by K. Tanaka and Y. Okuda at Kyoto University indicated no significant phonon peaks above $560 \mathrm{~cm}^{-1}$, equivalent to the energy of $800 \mathrm{~K}$.

${ }^{26}$ Y. Wang, T. Plackowski, and A. Junod, Physica C 355, 179 (2001).

${ }^{27}$ W. L. McMillan, Phys. Rev. 167, 331 (1968).

${ }^{28}$ R. P. Groff, A. Suna, and R. E. Merrifield, Phys. Rev. Lett. 33, 418 (1974).

${ }^{29}$ H. Gutfreund and M. Weger, Phys. Rev. B 16, 1753 (1977). 